\title{
Origen de los nervios del plexo braquial del venado coliblanco (Odocoileus virginianus) en comparación con otros rumiantes
}

\author{
ORIGIN OF THE BRACHIAL PLEXUS NERVES OF THE WHITE-TAILED DEER (Odocoileus \\ virginianus) IN COMPARISON WITH OTHER RUMINANTS \\ Juan Fernando Vélez García ${ }^{1,4}$, Alejandra Ospina Orozco², \\ Jorge Eduardo Duque Parra ${ }^{3}$
}

\section{Resumen}

El presente trabajo tuvo como objetivo identificar los nervios espinales que dan origen al plexo braquial y el origen de cada uno de los nervios del venado coliblanco (Odocoileus virginianus), así como comparar los resultados con lo reportado en la literatura para otros rumiantes. Se diseccionaron los dos plexos braquiales de cuatro especímenes (tres hembras y un macho). El plexo braquial se originó por contribución de las raíces de los ramos ventrales de los nervios espinales desde C6 a T1 (3/4), y desde C6 a T2 (1/4). De estos se originó el nervio supraescapular, subescapulares, axilar, radial, ulnar, mediano, toracodorsal, musculocutáneo, pectorales craneales, pectorales caudales, torácico largo, torácico lateral, subclavio y escapular dorsal. El origen del plexo braquial de $O$. virginianus fue más similar a lo reportado en el bovino. La formación de los nervios presenta similitudes y variantes con lo reportado en otros rumiantes. Así mismo, puede haber variaciones anatómicas interespecíficas, intraespecíficas e incluso en los plexos colaterales del mismo espécimen para el origen de los nervios.

Palabras clave: anatomía; cérvido; fauna silvestre; nervios espinales

\section{AbSTRACT}

The objective of this study was to identify the spinal nerves that give origin to the brachial plexus and the origin of each of the nerves of the white-tailed deer (Odocoileus virginianus), as well as to compare the results with those reported in the literature for

\footnotetext{
${ }^{1}$ Departamento de Sanidad Animal, Facultad de Medicina Veterinaria y Zootecnia, Universidad del Tolima, Ibagué, Tolima, Colombia

${ }^{2}$ Programa de Zootecnia, Corporación Universitaria Santa Rosa de Cabal (UNISARC), Santa Rosa de Cabal, Risaralda, Colombia

${ }^{3}$ Departamento de Ciencias Básicas, Facultad de Ciencias para la Salud, Universidad de Caldas, Manizales, Caldas, Colombia

${ }^{4}$ E-mail:jfvelezg@ut.edu.co
}

Recibido: 25 de diciembre de 2017

Aceptado para publicación: 20 de junio de 2018 
other ruminants. The two brachial plexuses of four specimens (three females and one male) were dissected. The brachial plexus was originated by contribution of the roots of the ventral branches of the spinal nerves from C6 to T1 (3/4), and from C6 to T2 (1/4). From these originated the suprascapular, subscapular, axillary, radial, ulnar, median, thoracodorsal, musculocutaneous, cranial pectoral, pectoral caudal, long thoracic, lateral thoracic, subclavian, and dorsal scapular nerves. The origin of the brachial plexus of $O$. virginianus was more similar to that reported in cattle. The formation of the nerves presents similarities and variants with what has been reported in other ruminants. Likewise, there may be interspecific, intraspecific anatomical variations, and even in the collateral plexuses of the same specimen for the origin of the nerves.

Key words: anatomy; cervid; wildlife; spinal nerves

\section{INTRODUCCIÓN}

El venado coliblanco (Odocoileus virginianus) es un cérvido distribuido desde el sur de Canadá hasta Brasil, generalmente hasta el norte del Amazonas (Smith, 1991). En Colombia se cuenta con cinco subespecies, entre ellas el Odocoileus virginianus curassavicus en la zona andina, valles y llanuras del norte de Colombia y Curazao (Brokx, 1984; López-Arévalo y González-Hernández, 2006).

Los ejemplares tropicales de venado coliblanco son de menor tamaño, con una longitud corporal de 1.2-1.5 m, los machos pesan cerca de $50 \mathrm{~kg}$ y las hembras cerca de $30 \mathrm{~kg}$ (Halls, 1984). Taxonómicamente se diferencia de las subespecies norteamericanas por cuatro rasgos principales: divergencia genética, cornamenta más pobre, ausencia de glándulas metatarsianas y en menor grado por el tamaño corporal (Molina y Molinari, 1999).

La mayoría de las poblaciones en México, Centro y Sur América están en descenso, y el estado de conservación de muchas subespecies es desconocido (Gallina y LópezArévalo, 2016). En general, las mayores amenazas a las que se enfrentan los cérvidos neotropicales es la pérdida de sus hábitats por el crecimiento de la frontera agrícola, la introducción de ganado (Duarte et al., 2001), la caza furtiva (Gallina y López-Arévalo,
2016) y muerte por atropello (Brinkman, 2003). Entre los traumas que pueden sufrir se incluyen aquellos que afectan el miembro torácico (Hattel et al., 2004; Phelps et al., 2010), al igual que en otras especies de venados (Quessada, 1993; Singh et al., 2010; Erdikmen et al., 2012), los cuales se convierten en candidatos para tratamientos ortopédicos más prácticos y diferentes a los que se realizan en animales de gran tamaño (Kaneps, 1996).

La parte somática del sistema nervioso hace posible la interacción del cuerpo con el ambiente externo e interno. Estas funciones se llevan a cabo en el miembro torácico mediante el plexo braquial, el cual se forma por la confluencia de los ramos ventrales de los tres últimos nervios cervicales y el primer nervio torácico (C6, C7, C8 y T1), y algunas veces con contribución del quinto nervio cervical (C5) y el segundo nervio torácico (T2) (Barone y Simoens, 2010; Dyce et al., 2012). En la práctica de anestesia, este plexo se puede ubicar mediante ultrasonido en el bovino (Iwamoto et al., 2012) o con un estimulador nervioso para aplicar anestésicos que bloqueen su conducción nerviosa en ovejas (Ghadirian y Vesal, 2013). Estas técnicas podrían ser empleadas en cérvidos bajo anestesia general, ya que esta y la cirugía en venados, es similar a la utilizada en los bovinos (Duarte et al., 2001). No obstante, un mejor conocimiento de la anatomía de cada especie permite establecer mejores procedimien- 
tos anestésicos regionales y quirúrgicos, pues toman en cuenta las variantes anatómicas.

Las subespecies sudamericanas del venado coliblanco no han sido tan estudiadas en comparación con las subespecies de Centro y Norte América (Weber y González, 2003). En el venado coliblanco se han realizado estudios anatómicos como la descripción de las arterias del hombro y brazo (Bisaillon, 1974), la anatomía gastrointestinal (Westerling, 1975; Jenks et al., 1994), la morfología craneana (Rees, 1969a,b, 1970; Molina y Molinari, 1999), las glándulas prepuciales (Odend'hal et al., 1992) y las vértebras cervicales (Solounias, 1999); sin embargo, son escasos los estudios sobre la anatomía del sistema nervioso, entre ellos, el plexo braquial. Por lo tanto, el presente trabajo tuvo como objetivo identificar los nervios espinales que dan origen al plexo braquial y el origen de cada uno de los nervios, así como comparar los resultados con lo reportado en la literatura para otros rumiantes.

\section{Materiales y Métodos}

Se disecaron los cadáveres de tres hembras y un macho de la especie Odocoileus virginianus provenientes de la Corporación Autónoma Regional de Caldas (CORPOCALDAS). Los especímenes llegaron para necropsia al hospital veterinario Diego Villegas Toro de la Universidad de Caldas. Los animales fueron identificados como de la subespecie Odocoileus virginianus curassavicus debido a la región geográfica de proveniencia.

Se realizó la necropsia teniendo precaución de no afectar los nervios de interés para esta investigación. Los miembros torácicos junto con el tronco de un macho y de dos hembras fueron fijados y conservados mediante la aplicación de inyecciones intramusculares y subcutáneas con una solución en mezcla de formaldehído al 10\%, aceite mineral al 5\% y ácido fénico al $1 \%$.
Posteriormente fueron sumergidos en la misma solución, pero sin aceite mineral. Luego de 48 horas de fijación se realizó la disección de superficial a profundo ventralmente en la región cervico-torácica hasta encontrar los forámenes intervertebrales. Se visualizó la contribución aparente de las raíces nerviosas de los ramos ventrales de los nervios espinales para la formación del plexo braquial y la contribución de cada uno para la formación de los nervios que se originan de este. La tercera hembra fue congelada posterior a la necropsia y en fecha posterior se diseccionó en fresco. Para la descripción anatómica se tuvieron en cuenta los términos aceptados en la Nómina Anatómica Veterinaria (ICVGAN, 2017).

\section{Resultados}

El plexo braquial de los dos miembros locomotores torácicos de un espécimen se originó en C6, C7, C8 y T1 (C6-T1) (Figura 1), mientras que en los otros tres especímenes se originó de las raíces nerviosas de los ramos ventrales de los nervios espinales de C6, $\mathrm{C} 7, \mathrm{C} 8, \mathrm{~T} 1$ y un débil ramo proveniente de T2 (C6-T2) (Figura 2).

De los ocho plexos diseccionados se encontraron la formación de los siguientes nervios: el nervio subclavio (N. subclavius) de $\mathrm{C} 7$ y el nervio escapular dorsal ( $N$. dorsalis scapulae) de C6, el nervio supraescapular ( $N$. suprascapularis) por C6 y $\mathrm{C} 7$, los nervios subescapulares ( $\mathrm{Nn}$. subscapulares) por C6 y C7, el nervio torácico largo ( $N$. thoracicus longus) por $\mathrm{C} 7 \mathrm{y} \mathrm{C} 8$, los nervios pectorales caudales $(\mathrm{Nn}$. pectorales caudales) por $\mathrm{C} 8$ y $\mathrm{T} 1$, el nervio radial ( $N$. radialis) por $\mathrm{C} 7, \mathrm{C} 8$ y $\mathrm{T} 1$, el nervio mediano por $\mathrm{C} 7, \mathrm{C} 8$ y T1, donde la contribución de $\mathrm{C} 7$ fue a nivel del asa axilar a través del nervio musculocutáneo.

En los siguientes nervios, la formación no fue similar en todos los animales: el nervio musculocutáneo (N. musculocutaneus) for- 


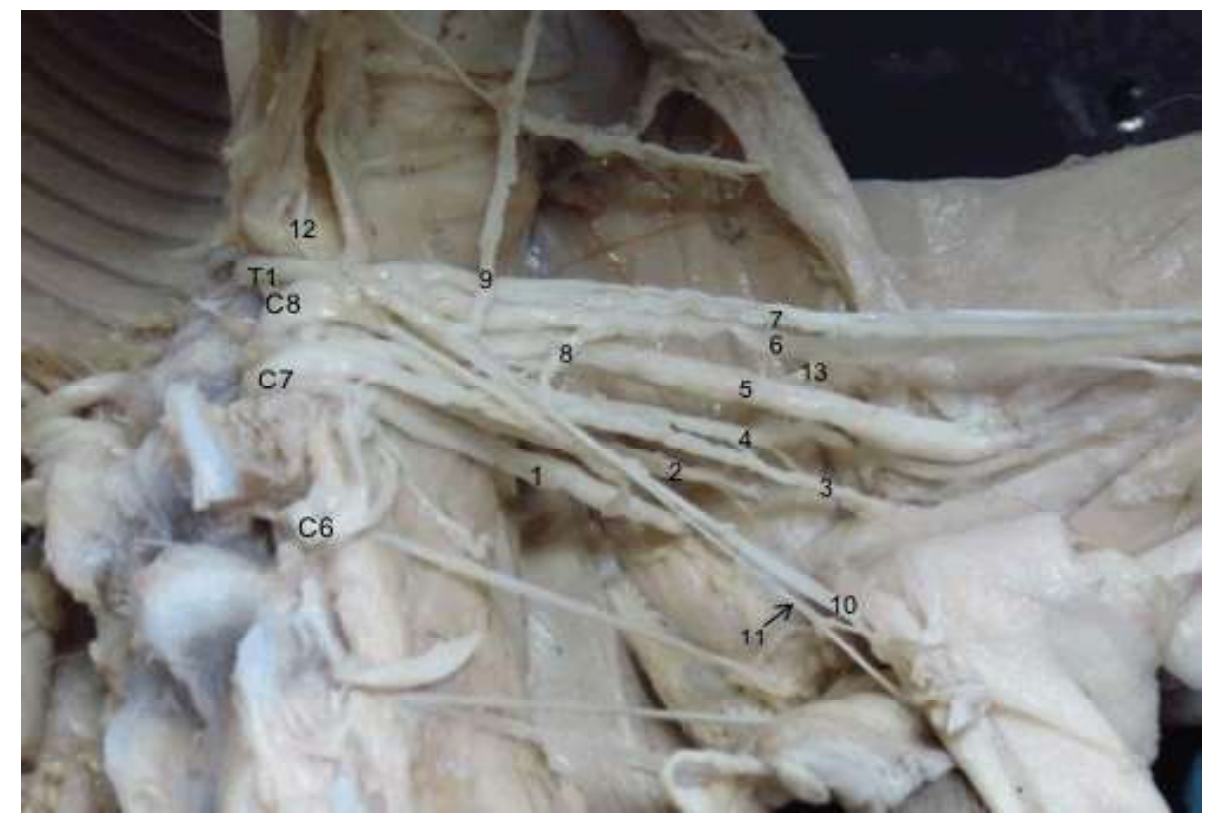

Figura 1. Vista ventral del plexo braquial del venado coliblanco (Odocoileus virginianus) formado de C6-T1. C: Nervio espinal cervical; T: Nervio espinal torácico; $1: \mathrm{N}$. supraescapular; 2: Nn. Subescapulares; 3: N. musculocutáneo; 4: N. axilar; 5: N. radial; 6: N. mediano; 7: N. ulnar; 8: asa axilar; 9: N. torácico lateral; 10: Nn. pectorales caudales; 11: Nn. pectorales craneales; 12: N. torácico largo; 13: N. toracodorsal

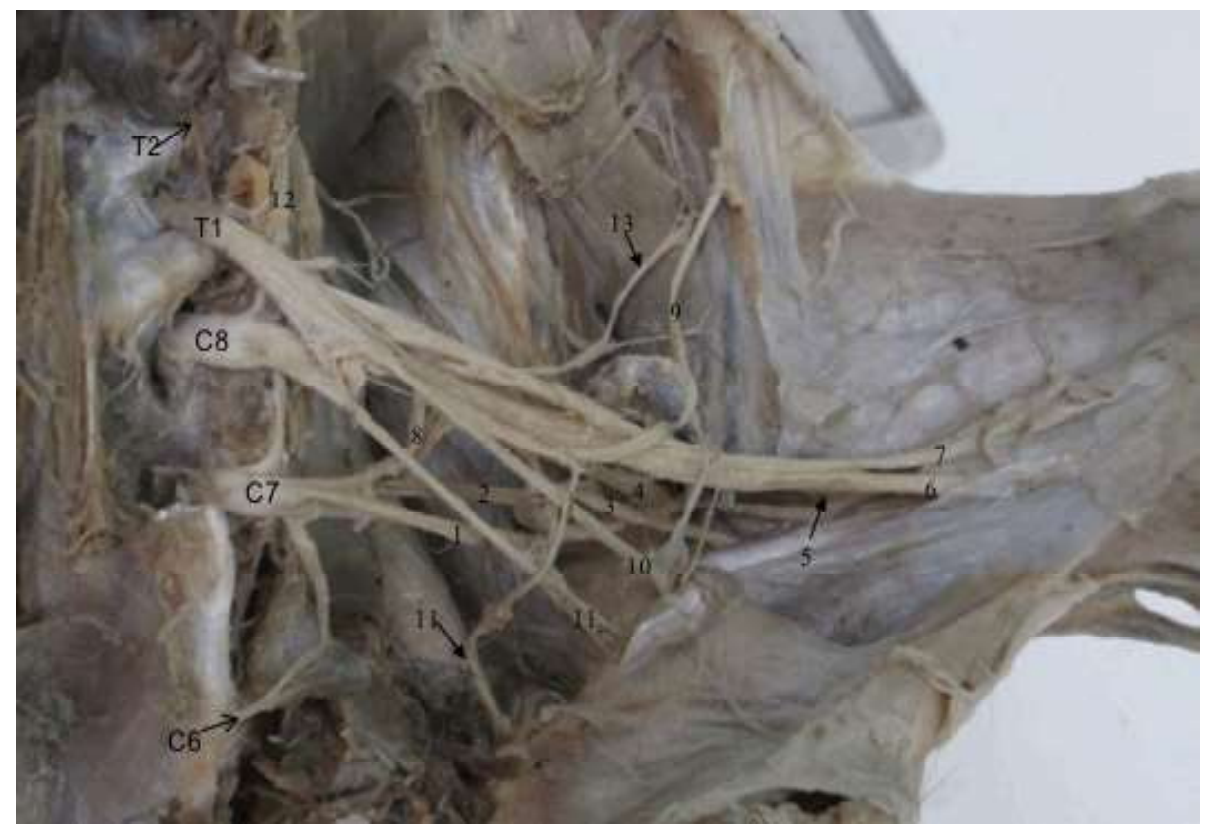

Figura 2. Vista ventral del plexo braquial del venado coliblanco (Odocoileus virginianus) formado de C6-T2. C: Nervio espinal cervical; T: Nervio espinal torácico; 1: N. supraescapular; 2: Nn. Subescapulares; 3: N. musculocutáneo; 4: N. axilar; 5: N. radial; 6: N. mediano; 7: N. ulnar; 8: asa axilar; 9: N. torácico lateral; 10: Nn. pectorales caudales; 11: Nn. pectorales craneales; 12: N. torácico largo; 13: N. toracodorsal 
Cuadro 1. Origen de los nervios del plexo braquial del venado coliblanca (Odocoileus virginianus)

\begin{tabular}{|c|c|c|c|c|c|c|c|c|}
\hline \multirow{2}{*}{ Nervio } & \multicolumn{2}{|c|}{ Espécimen 1} & \multicolumn{2}{|c|}{ Espécimen 2} & \multicolumn{2}{|c|}{ Espécimen 3} & \multicolumn{2}{|c|}{ Espécimen 4} \\
\hline & MTD & MTI & MTD & MTI & MTD & MTI & MTD & MTI \\
\hline $\begin{array}{l}\text { Origen del } \\
\text { plexo braquial }\end{array}$ & C6-T1 & C6-T1 & C6-T2 & C6-T2 & C6-T2 & C6-T2 & C6-T2 & C6-T2 \\
\hline $\begin{array}{l}\text { Pectorales } \\
\text { caudales }\end{array}$ & $\mathrm{C} 8-\mathrm{T} 1$ & $\mathrm{C} 8-\mathrm{T} 1$ & C8-T1 & $\mathrm{C} 8-\mathrm{T} 1$ & C8-T1 & $\mathrm{C} 8-\mathrm{T} 1$ & C8-T1 & $\mathrm{C} 8-\mathrm{T} 1$ \\
\hline $\begin{array}{l}\text { Pector } \\
\text { cran }\end{array}$ & $\mathrm{C} 7-\mathrm{C} 8$ & $\mathrm{C} 7-\mathrm{C} 8$ & $\mathrm{C} 7-\mathrm{C} 8$ & $\mathrm{C} 7-\mathrm{C} 8$ & C7-C8 & C7-C8 & C7-T1 & C7-T1 \\
\hline & C8-T1 & C8-T1 & C8-T2 & $\mathrm{C} 8-\mathrm{T} 2$ & $\mathrm{C} 8-\mathrm{T} 2$ & C8-T1 & C8-T1 & C8-T1 \\
\hline & $\mathrm{C} 7-\mathrm{C} 8$ & $\mathrm{C} 7-\mathrm{C} 8$ & $\mathrm{C} 7-\mathrm{C} 8$ & C8 & C8-T1 & C7-T1 & C8-T1 & $\mathrm{C} 8-\mathrm{T} 1$ \\
\hline & $\mathrm{C} 7-\mathrm{C} 8$ & $\mathrm{C} 7-\mathrm{C} 8$ & $\mathrm{C} 7-\mathrm{C} 8$ & $\mathrm{C} 7-\mathrm{C} 8$ & $\mathrm{C} 7-\mathrm{C} 8$ & $\mathrm{C} 7-\mathrm{C} 8$ & $\mathrm{C} 7-\mathrm{C} 8$ & $\mathrm{C} 7-\mathrm{C} 8$ \\
\hline & C6-C7 & $\mathrm{C} 6-\mathrm{C} 7$ & C6-C7 & C6-C7 & C6-C7 & $\mathrm{C} 6-\mathrm{C} 7$ & C6-C7 & C6-C7 \\
\hline & C6-C7 & C6-C7 & C6-C7 & $\mathrm{C} 6-\mathrm{C} 7$ & C6-C7 & C6-C7 & C6-C7 & C6-C7 \\
\hline Axilar & C6-C8 & C6-C8 & C6-C8 & $\mathrm{C} 6-\mathrm{C} 7$ & C6-C8 & C6-C8 & C6-C8 & C6-C8 \\
\hline & C7-T1 & C7-T1 & C7-T1 & C7-T1 & C7-T1 & C7-T1 & C7-T1 & C7-T1 \\
\hline Ulnar & $\mathrm{C} 8-\mathrm{T} 1$ & $\mathrm{C} 8-\mathrm{T} 1$ & $\mathrm{C} 8-\mathrm{T} 2$ & $\mathrm{C} 8-\mathrm{T} 2$ & $\mathrm{C} 8-\mathrm{T} 2$ & $\mathrm{C} 8-\mathrm{T} 1$ & C8-T1 & C8-T1 \\
\hline & C7-T1 & C7-T1 & C7-T1 & C7-T1 & C7-T1 & C7-T1 & C7-T1 & C7-T1 \\
\hline Musculo & C6-C8 & C6-C8 & C6-C8 & C6-C7 & C6-C7 & C6-C8 & C6-C8 & $\mathrm{C6}-\mathrm{C} 8$ \\
\hline & $\mathrm{C} 7$ & $\mathrm{C} 7$ & $\mathrm{C} 7$ & $\mathrm{C} 7$ & C7 & $\mathrm{C} 7$ & $\mathrm{C} 7$ & C7 \\
\hline Escapular dorsal & C6 & C6 & C6 & C6 & C6 & C6 & C6 & C6 \\
\hline
\end{tabular}

C: Nervio espinal cervical; T: Nervio espinal torácico

MTD: miembro torácico derecho; MTI: miembro torácico izquierdo

mado por contribución de C6-C7-C8 en 3 de los 4 casos. La contribución de $\mathrm{C} 8$ proviene a nivel del asa axilar por ramos comunicantes del nervio mediano ( $N$. medianus); diferente a lo encontrado en uno de los especímenes, donde, si bien se encontró la formación del asa axilar, no se observaron ramos comunicantes provenientes del nervio mediano, siendo su origen solo de C6 y C7. El nervio axilar ( $N$. axillaris) fue formado por C6, C7 y $\mathrm{C} 8$ en 7 de los 8 miembros y solo de C6-C7 en un miembro. Los nervios pectorales craneales $(\mathrm{Nn}$. pectorales craniales) fueron formados por $\mathrm{C} 7 \mathrm{y} \mathrm{C} 8$ en el 3 de 4 especímenes, mientras que en el otro recibieron contribuciones de T1 (C7-T1). El nervio toracodorsal ( $N$. thoracodorsalis) fue formado por $\mathrm{C} 7$ y $\mathrm{C} 8$ en tres miembros, de $\mathrm{C} 8$ y $\mathrm{T} 1$ en otros tres miembros, de $\mathrm{C} 7$, $\mathrm{C} 8$, y $\mathrm{T} 1$ en un miembro y solo de $\mathrm{C} 8$ en un miembro. El nervio torácico lateral $(N$. thoracicus lateralis) fue formado por $\mathrm{C} 8$, T1 y T2 en 3 de 4 especímenes y sólo de C8T1 en el otro espécimen. El nervio ulnar $(N$. ulnaris) fue formado por $\mathrm{C} 8, \mathrm{~T} 1, \mathrm{y}$ T2 en 3 de 4 especímenes y solo desde C8-T1 en el otro espécimen (Cuadro 1).

\section{Discusión}

Las raíces del plexo braquial en el bovino provienen de los ramos ventrales de $\mathrm{C} 6$ a T2 (Magilton, 1966; Ghoshal, 1982; Budras et al., 2011, Dyce et al., 2012), siendo similar a lo encontrado en la mayoría de especímenes 
de $O$. virginianus, aunque uno presentó origen de C6-T1, similar al guazuncho (Mazama gouazoubira) (Melo et al., 2007, Vieira et al., 2013), okapi (Okapia johnstoni) (Endo et al., 2009), serau japonés (Capricornis crispus) (Atoji et al., 1987), ovinos y caprinos (May 1974; Ghoshal 1982; Karim et al., 2008; Barone y Simoens, 2010), aunque en estos tres últimos pueden haber casos excepcionales donde hay contribución de T2 (Barone y Simoens, 2010), lo cual también puede acontecer en el bovino (Barone y Simoens, 2010) y en $O$. virginianus.

El nervio supraescapular en $O$. virginianus presenta un origen desde C6-C7, como ocurre en los rumiantes domésticos (Magilton, 1966; May, 1974; Ghoshal, 1982; Barone y Simoens, 2010, Budras et al., 2011; Dyce et al., 2012), O. johnstoni (Endo et al., 2009) у M. gouazoubira (Melo et al., 2007; Vieira et al., 2013), aunque en esta última especie lo reportan originado solo de C6 en hembras (Melo et al., 2007). En caprinos se ha encontrado formado tan solo por C6 en un plexo braquial (Magilton, 1966).

Los nervios subescapulares se pueden originar únicamente desde $\mathrm{C} 7$ en ovinos, caprinos (Magilton, 1966; Ghoshal, 1982), O. johnstoni (Endo et al., 2009), en hembras de M. gouazoubira (Melo et al., 2007), pero no en $O$. viriginianus donde siempre tuvieron contribución de C6, similar al bovino (Magilton, 1966; May, 1974; Ghoshal, 1982; Barone y Simoens, 2010). También puede haber contribución de C6 en caprinos y ovinos (Magilton, 1966) y en M. gouazoubira (Vieira et al., 2013), e incluso de C8 en bovinos (Barone y Simoens, 2010; Budras et al., 2011).

El nervio musculocutáneo en $O$. virginianus se origina igual que en el patrón común del bovino de C6-C8 (Magilton, 1966; Ghoshal, 1982; Budras et al., 2011), aunque se describe que en todos los rumiantes domésticos se origina de C6-C7 (ovinos: Magilton [1966], May [1974], Ghoshal [1982], caprinos: Magilton [1966], Ghoshal [1982], M. gouazoubira: Melo et al. [2007], Vieira et al. [2013]), lo cual se presentó como variante en $O$. virginianus. En esta especie se ha encontrado originando, además, de C7-C8 (Endo et al., 2009), el cual puede ser un origen variante en ovinos (Magilton, 1966; Ghoshal, 1982) y en bovinos (Magilton, 1966), pero no fue hallado en $O$. virginianus. En ovinos también se puede encontrar como variante originado de C6-C8 (Magilton, 1966).

La contribución para los nervios musculocutáneo, mediano y ulnar en el serau japonés (Capricornis crispus) puede variar de acuerdo a los tipos de troncos formados por C6-T1. En la mayoría de los casos, los cuatro ramos forman un tronco para los tres nervios (el musculocutáneo es representado por dos ramos musculares: uno proximal y uno distal). En el segundo tipo, los cuatro nervios formaron el nervio musculocutáneo y mediano juntos, en el tercer caso el ulnar se originó de solo C8 y T1, y en el cuarto caso se formó un tronco común para los tres nervios de C8 y T1; aunque también puede haber de $\mathrm{T} 2$, aunque es una rara variación (1 de 26) (Atoji et al., 1987).

El nervio axilar se originó principalmente de C6-C8, el cual es un origen que se puede presentar en menor proporción en ovinos (Magilton, 1966; May, 1974; Ghoshal, 1982); sin embargo, en $O$. virginianus también puede faltar C8 como en M. gouazoubira (Vieira et al., 2013), ya que en esta última especie se ha encontrado en mayor proporción originado en machos de C6-T1 y en hembras de C7-T1 (Melo et al., 2007). Esta ausencia se encuentra en mayor proporción en ovinos y caprinos (Magilton, 1966; Ghoshal, 1982; Barone y Simoens, 2010). Otros orígenes reportados no fueron hallados en $O$ virginianus, como el C7-C8 en el patrón común de bovinos (Magilton, 1966; Ghoshal, 1982; Barone y Simoens, 2010; Budras et al., 2011) y como se puede presentar en $O$. johnstoni (Endo et al., 2009) y variante en ovinos (Magilton, 1966). 
El nervio radial se originó de C7-T1, al igual que en rumiantes domésticos (Magilton, 1966; May, 1974; Ghashol, 1982; Budras et al., 2011; Dyce et al., 2012) y en $O$. johnstoni (Endo et al., 2009). No obstante, en rumiantes domésticos también se observa contribución de C6 (Barone y Simoens, 2010), como sucede en machos de M. gouazoubira a diferencia de las hembras.

El nervio mediano presentó igual origen (C7-T1) que lo reportado en M. gouazoubira (Vieira et al., 2013), aunque en esta última también se puede encontrar originado de C8$\mathrm{T} 1$ en machos y tan solo de T1 en hembras (Melo et al., 2007). Por otro lado, el $O$. virginianus se diferencia de otros rumiantes, porque en bovinos se origina de C8-T2 (Magilton, 1966; Ghashol, 1982; Budras et al., 2011; Dyce et al., 2012), en ovinos y caprinos de C8-T1 (Magilton, 1966; May, 1974; Ghashol, 1982) e incluso la contribución de T2 es eventual en todos los mamíferos domésticos (Barone y Simoens, 2010). En $O$. johnstoni se encuentra originado solo de $\mathrm{T} 1$ (Endo et al., 2009), siendo diferente a la mayoría de los rumiantes antes descritos.

El nervio ulnar se originó igual que en bovinos de C8-T2 (Magilton, 1966; Ghashol, 1982; Budras et al., 2011; Dyce et al., 2012), y también en menor proporción no hubo contribución de T2 similar a lo que puede ocurrir en rumiantes domésticos (Barone y Simoens, 2010), principalmente en ovinos y caprinos donde la contribución de T2 para la formación del plexo braquial es excepcional (Magilton, 1966; May, 1974; Ghashol, 1982), al igual que en C. crispus (Atoji et al., 1987) y en M. gouazoubira (Melo et al,. 2007; Vieira et al., 2013). En esta última especie se puede encontrar originado solamente desde T1 en hembras (Melo et al., 2007) al igual que en $O$. johnstoni (Endo et al., 2009).

Los nervios pectorales craneales se originaron de $\mathrm{C} 7-\mathrm{C} 8$, similar a lo que sucede en rumiantes domésticos, aunque en caprinos se puede encontrar como variante originado solo de C8 o de C6-C8 (Magilton, 1966). En ru- miantes domésticos se reporta que se pueden originar de C6 y C7, y a veces de C8 (Barone y Simoens, 2010). En el ovino se reportan originados de $\mathrm{C} 8$ y $\mathrm{T} 1$, junto con el nervio mediano o nervio el torácico lateral (May, 1974). En el caprino también se puede originar de C6-C7, algunas veces con contribución única de C7 (Magilton, 1966). En $M$. gouazoubira los reportan como ramificaciones del tronco común del nervio mediano y ulnar, el cual se forma de C8-T1 en machos y solo de T1 en hembras (Melo et al., 2007). La contribución adicional de T1 se presentó como una variante en $O$. virginianus.

Los nervios pectorales caudales se originaron de C8-T1 igual que en el ovino junto con el nervio torácico lateral (May, 1974), aunque se reporta que en todos los rumiantes domésticos la contribución es de C7-T1 (Barone y Simoens, 2010) y en $M$. gouazoubira los reportan como ramificaciones del nervio torácico largo en machos y del nervio torácico lateral en hembras (Melo et al., 2007). En caprinos solo se origina desde C8 (Magilton, 1966).

El nervio toracodorsal presentó orígenes diversos, presentándose de C7-C8 como sucede en bovinos (Ghashol, 1982; Budras et al., 2011) y en M. gouazoubira (Vieira et al., 2013) como variante en ovinos (Magilton, 1966); de C8-T1 como variante en bovinos (Magilton, 1966) y ovinos (May, 1974), aunque en este último se ha encontrado en mayor proporción solo de C8 (Magilton, 1966; Ghashol, 1982); en cambio, en O. virginianus fue encontrada como una variante. En $M$. gouazoubira se puede llegar a originar desde C6-T1 en machos y de C8-T1 en hembras (Melo et al., 2007). En bovinos también se le puede encontrar originado entre C7-T1 (Magilton, 1966).

El nervio torácico lateral se originó en mayor proporción de C8-T2, al igual que en el bovino (Magilton, 1966; Ghashol, 1982; Budras et al., 2011); por otro lado, en un espécimen de $O$. virginianus se originó solo desde C8-T1, como sucede en ovinos y 
caprinos (Magilton, 1966; May, 1974; Ghashol, 1982) y en M. gouazoubira (Vieira et al., 2013), o como una variante en bovinos (Magilton, 1966; Ghashol, 1982). En M. gouazoubira se ha reportado originado de C6-T1 en machos y solo desde T1 en hembras (Melo et al., 2007), donde esto último se puede presentar en $O$. johnstoni (Endo et al., 2009).

El nervio torácico largo presentó origen de C7-C8, al igual que en rumiantes domésticos (Magilton, 1966; May, 1974; Ghashol, 1982; Budras et al., 2011) y en $M$. gouazoubira (Vieira et al., 2013), aunque en esta última especie también se encuentra originado solo de $\mathrm{C} 7$ en machos y de $\mathrm{T} 1$ en hembras (Melo et al., 2007).

El nervio subclavio solo se originó de C7, a diferencia de rumiantes domésticos, donde se origina de C6 y C7 (Barone y Simoens, 2010). El nervio escapular dorsal se originó de C6, similar a lo reportado en rumiantes domésticos (Barone y Simoens, 2010).

\section{Conclusiones}

- El plexo braquial del Odocoileus virginianus se originó en mayor proporción de C6-T2, conservando una mayor similitud con el bovino, aunque también puede faltar la contribución de T2, como ocurre en el patrón común de ovinos y caprinos.

- En el origen de los nervios del $O$. virginianus se pueden encontrar variantes interespecíficas, intraespecíficas y en los plexos colaterales de un mismo espécimen, las cuales deben ser tenidas en cuenta para procedimientos veterinarios.

\section{Literatura Citada}

1. Atoji Y, Suzuki Y, Sugimura M. 1987. The brachial plexus of the Japanese serow (Capricornis crispus). Anat Anzeiger 163: 25-32.
2. Barone R, Simoens P. 2010. Anatomie comparée des mammifères domestiques. Neurologie II. Système nerveux périphérique, glandes endocrines, esthésiologie. Vigot. $1021 \mathrm{p}$.

3. Bisaillon A. 1974. The major arteries of the shoulder and arm of the white tailed deer (Odocoileus virginianus, Rafinesque, 1832). Anat Histol Embryol 3: 250-261. doi: $10.1111 / \mathrm{j} .1439$ 0264.1974.-tb00618.x

4. Brinkman TJ. 2003. Movement and mortality of white-tailed deer in southwest Minnesota. MSc Thesis. USA: South Dakota State University. 135 p.

5. Brokx PA. 1984. South America. In: Halls KL (ed). White-tailed deer. Ecology and management. Pennsylvania, USA: Strackpole Books. p 525-546.

6. Budras KD, Habel RE, Mülling $C$, Greenough P. 2011. Bovine anatomy: an illustrated text. $2^{\text {nd }}$ ed. Germany: Schlütersche. $176 \mathrm{p}$.

7. Duarte JM, Merino M, Gonzalez S, Nunes AL, Garcia J, Szabó M, Pandolfi JR., et al. 2001. Order Artiodactyla, Family Cervidae (Deer). In: Fowler ME, Cubas Z (eds). Biology, medicine, and surgery of South American wild animals. Iowa, USA: Iowa State University Press. p 402-422.

8. Dyce KM, Sack WO, Wensing CJG. 2012. Anatomía veterinaria. $4^{\circ}$ ed. México: El Manual Moderno. 833 p.

9. Endo H, Koyabu D, Hayashida A, Oishi M, Kawada SI, Komiya T. 2009. The brachial plexus adapted to the semielongated neck in the okapi. Mamm Study 34: 209-212. doi: 10.3106/ 041.034 .0405

10. Erdikmen DO, Özsoy S, Aydin D. 2012. Partially forelimb amputation and application of an artificial limb (prosthetics) in a free-ranging red deer (Cervus elaphus). Kafkas Univ Vet Fak 18:347-350. doi: 10.9775/kvfd.2011.5721

11. Gallina S, López-Arevalo H. 2016. Odocoileus virginianus. The IUCN Red List of Threatened Species 2016: [Internet]. Disponible en: http:// 
dx.doi.org/10.2305/IUCN.UK.2016-

2.RLTS.T42394A22162580.en

12. Ghadirian S, Vesal N. 2013. Brachial plexus block using lidocaine/epinephrine or lidocaine/xylazine in fat-tailed sheep. Vet Res Forum 4: 161-167.

13. Ghoshal NG. 1982. Sistema nervioso de los rumiantes: nervios espinales. En: Getty R (ed). Anatomía de los animales domésticos de Sisson y Grossman. $5^{\circ}$ ed. Barcelona: Masson. p 1242-1269.

14. Halls LK. 1984. White-tailed deer: ecology and management. Washintong DC: Stackpole Books. 870 p.

15. Hattel AL, Shaw DP, Love BC, Wagner DC, Drake, TR, Brooks JW. 2004. A retrospective study of mortality in Pennsylvania captive white-tailed deer (Odocoileus virginianus): 2000-2003. J Vet Diagn Invest 16: 515-521. doi: 10.1177/104063870401600605

16. [ICVGAN] International Committee on Veterinary Gross Anatomical Nomenclature. 2017. Nomina Anatomica Veterinaria. $5^{\circ}$ ed. Hannover, Germany: ICVGAN. 178 p.

17. Iwamoto J, Yamagishi N, Sasaki K, Kim D, Devkota B, Furuhama K. 2012. A novel technique of ultrasoundguided brachial plexus block in calves. Res Vet Sci 93: 1467-1471. doi: 10.1016/ j.rvsc. 2012.05.010

18. Jenks JA, Leslie Jr DM, Lochmiller RL, Melchiors MA. 1994. Variation in gastrointestinal characteristics of male and female white-tailed deer: implications for resource partitioning. J Mammal 75: 1045-1053. doi: $10.2307 / 1382488$

19. Kaneps AJ. 1996. Orthopedic conditions of small ruminants: llama, sheep, goat, and deer. Vet Clin N Am-Food A 12:211-231.

20. Karim MR, Haque Z, Khan M, Akther SH, Gofur R. 2008. The brachial plexus of the black bengal goat (Capra hircus). Bangladesh J Prog Sci Tech 6: 113-116.

21. López-Arévalo H, GonzálezHernández A. 2006. Venado sabanero Odocoileus virginianus. En: Libro rojo de los mamíferos de Colombia. Se- rie libros rojos de especies amenazadas de Colombia, Bogotá: Conservación Internacional \& Ministerio de Ambiente y Desarrollo Territorial. p 114-120.

22. Magilton JH. 1966. A comparative morphological study of the brachial plexus of domestic animals (Bovidae, Ovidae, Capridae, Suidae, Equidae). PhD Thesis. USA: Iowa State University. $238 \mathrm{p}$.

23. May N. 1974. Anatomía del ovino: manual de disección. Argentina: Hemisferio sur. $561 \mathrm{p}$.

24. Melo SR, Gonçalves AF, de Castro TH, Fioretto ET, Gerbasi SH, Machado MR, et al. 2007. Sex-related macrostructural organization of the deer's brachial plexus. Anat Histol Embryol 36:295-299. doi: 10.1111/j.14390264.2007.00765.x

25. Molina M, Molinari J. 1999. Taxonomy of Venezuelan white-tailed deer(Odocoileus, Cervidae, Mammalia), based on cranial and mandibular traits. Can J Zool 77: 632-645. doi: 10.1139/ z98-235

26. Odend'hal S, Miller KV, Hoffmann DM. 1992. Preputial glands in the whitetailed deer (Odocoileus virginianus). J Mammal 73: 299-302. doi: 10.2307/ 1382060

27. Paterson AM. 1887. The limb plexuses of mammals. J Anat Physiol 21: 611-634.

28. Phelps HA, Lewis DD, Aiken-Palmer C, Winter MD. 2010. Use of a linearcircular hybrid external skeletal fixator for stabilization of a juxta-physeal proximal radial fracture in a deer (Odocoileus virginianus). J Zoo Wildlife Med 41: 688-696. doi: 10.1638/ 2009-0114.1

29. Quessada AM. 1993. Forelimb amputation in a red deer. Can Vet J 34: 445-446.

30. Rees JW. 1969a. Morphologic variation in the mandible of the white tailed deer (Odocoileus virginianus): a study of populational skeletal variation by principal component and canonical analyses. J Morphol 128: 113-130. doi: 10.1002/ jmor.1051280106 
31. Rees JW. 1969b. Morphologic variation in the cranium and mandible of the white tailed deer (Odocoileus virginianus): a comparative study of geographical and four biological distances. J Morphol 128: 95-112. doi: 10.1002/jmor.1051280105

32. Rees JW. 1970. A multivariate morphometric analysis of divergence in skull morphology among geographically contiguous and isolated groups of whitetailed deer (Odocoileus virginianus) in Michigan. Evolution 24: 220-229. doi: 10.1111/j.1558-5646.1970.tb01755.x

33. Singh K, Kumar A, Mahajan SK, Saini NS. 2010. Successful forelimb amputation procedure on a sambar deer (Cervus unicolor niger). J Wildlife Rehabil 30: 21-24.

34. Smith WP. 1991. Odocoileus virginianus. Mammalian species 388: 1-13. doi: $10.2307 / 3504281$
35. Solounias N. 1999. The remarkable anatomy of the giraffe's neck. J Zool 247: 257-268. doi: 10.1111/j.14697998.1999.tb00989.x

36. Vieira L, Ribeiro P, Lima M, Souza R, Valdes S, Santos A. 2013. Origens e ramificações do plexo braquial do veado catingueiro Mazama gouazoubira (Artiodactyla: Cervidae). Biotemas 26: 137-146 doi: 10.5007/2175-7925.2013v26n1p137

37. Weber M, Gonzalez S. 2003. Latin American deer diversity and conservation: a review of status and distribution. Ecoscience 10: 443-454. doi: 10.1080/ 11956860.2003.11682792

38. Westerling B. 1975. A comparative study of the intestinal anatomy of deer. Anat Anzeiger 137: 178-186. 